\title{
Genotoxicity of nanomaterials in vitro: treasure or trash?
}

\author{
Andrea Haase $^{1} \cdot$ Andreas Luch $^{1}$
}

Received: 16 November 2015 / Accepted: 22 August 2016

(C) Springer-Verlag Berlin Heidelberg 2016

Nanomaterials are increasingly used in a variety of applications, and plenty of nanoenabled products are already placed on the market. Nanomaterials often display novel and enhanced features compared to conventional materials. This led to an ongoing debate about the safety of these materials. Often it was concluded that the data pool is incomplete and insufficient to perform meaningful and reliable risk assessments for most of the nanomaterials. Additionally, it was questioned whether the established test methods for chemicals were at all adequate and suitable to assess the safety of nanomaterials. Has this situation changed meanwhile? In this editorial, we want to discuss the genotoxicity testing of nanomaterials by focusing on in vitro methodologies.

Genotoxicity describes the property of a chemical compound or of particles to alter the genetic information of a cell. This may occur by different mechanisms like gene mutations and structural or numerical chromosomal aberrations. In the following, such alterations can have severe consequences. In the germline, they can lead to abortions or to heritable damage to the offspring; in somatic cells, they may cause cancer. Furthermore, aging and a variety of diseases such cardiovascular or some neurodegenerative diseases are linked to the accumulation of DNA damage. Thus, genetic alterations are associated with serious health effects and may, in principle, occur even at very low exposure levels against the chemical culprit. Investigating the genotoxic properties therefore represents an important step in the safety assessment of a substance and is required

Andreas Luch

Andreas.Luch@bfr.bund.de

1 Department Chemical and Product Safety, German Federal Institute for Risk Assessment, Berlin, Germany under regulatory frameworks such as European REACH ("Registration, Evaluation, Authorisation and Restriction of Chemicals") or CLP ("Classification, Labelling and Packaging") regulation, but also in accordance with more specific directives such as those for cosmetics, foods or biocides.

State of the art of genotoxicity testing of chemicals is the application of a battery of assays covering gene mutations, structural and numerical chromosomal aberrations. At tier-1, this typically starts with an in vitro testing battery. If a chemical is without any effect in vitro, it might be classified as non-genotoxic in some legislations (REACH, CLP, Cosmetics Directive), while others (i.e., pharmaceuticals, veterinary drugs) usually require an in vivo follow-up investigation. On the other side, a positive outcome in vitro may also call for confirmation by appropriate in vivo testing. Yet, in recent years, in vivo studies in principle became under pressure or even prohibited under certain regulations such as the Cosmetics Directive (Adler et al. 2011).

Established in vitro gene mutation assays are the bacterial reverse mutation test, also referred to as Ames test (OECD TG 471) and the mammalian gene mutation tests (OECD TG 476 and 490), of which the latter two have been revised in 2015. The in vitro mammalian chromosomal aberration test, also referred to as CAT assay (OECD TG $473)$, is an established measure for the frequency of chromosomal aberrations. In addition, the in vitro mammalian cell micronucleus test, i.e., MNT (OECD TG 487), is supposed to detect structural and numerical aberrations and was only recently assessed by the European Centre for the Validation of Alternative Methods (ECVAM; Corvi et al. 2008); the test guideline has been revised thereafter. The MNT was considered less expensive and time consuming, thereby requiring less intense training and allowing a greater statistical validity compared to the CAT. Thus, the 
MNT was fastly included into REACH legislation to be used in the tier-1 genotoxicity testing battery. Other in vitro methods are currently not accepted for regulatory purposes.

Notwithstanding the biggest drawback of in vitro genotoxicity testing is the high rate of false positive results, which either triggers tremendous costs for in vivo followups or simply leads to the rejection of substances wrongly incriminated as genotoxicants. The high rate of false positives was discussed in an ECVAM workshop in April 2006, which evaluated options on how current in vitro genotoxicity tests can be improved and which new tests might be implemented in the future (Kirkland et al. 2007). After the workshop an expert group, coordinated by ECVAM, published lists of genotoxic and non-genotoxic chemicals to be used in performance checks aimed at advancing genotoxicity testing and facilitating the validation of new test methods (Kirkland et al. 2008). As another consequence of the workshop, several international initiatives were launched to develop and validate novel in vitro genotoxicity testing methods. For instance, the Japanese Centre for the Validation of Alternative Methods (JaCVAM) coordinated an initiative for validation of the comet assay in vivo and in vitro. Unfortunately, this only resulted in a novel test guideline for in vivo testing (OECD TG 489) in April 2014. In addition, Cosmetics Europe started an initiative to establish new methods for genotoxicity testing using the micronucleus and comet assay in human three-dimensional (3D) models (Aardema et al. 2010; Reus et al. 2013). The comet assay performed in human 3D models is currently running through the phase of validation.

To consider more realistic systems such as human 3D models or primary cells instead of cell lines, which may better reflect the metabolic capacity of the target organ(s), is one way of improving in vitro genotoxicity testing. Another would be the application of meaningful test concentrations. Particularly for in vitro tests, there is a tendency to use extremely high test concentrations of the chemicals, a factor known to contribute to the high false positive rates. The issue of test concentrations was also intensely discussed during guideline revisions at the level of the International Council for Harmonisation of Technical Requirements for Pharmaceuticals for Human Use (ICH) and the Organisation for Economic Co-operation and Development (OECD), and it was argued that maximum concentrations can be easily reduced without any loss of sensitivity (Parry et al. 2010).

In particular, genotoxicity testing of nanomaterials has gained increasing importance in recent years. Nanomaterials are not expected to induce as yet unknown kinds of genotoxic damage beyond that range already known for chemicals (i.e., gene mutations, structural or numerical chromosomal aberrations). Therefore, one might assume that the principles of genotoxicity testing, well established for chemicals, should also be applicable in the case of nanomaterials (Oesch and Landsiedel 2012; Pfuhler et al. 2013). At the OECD level, the guidelines for genotoxicity testing have been assessed for their suitability to assess nanomaterials. An OECD workshop focusing on the genotoxicity of manufactured nanomaterials has been organized in 2013 (ENV/JM/MONO(2014)34), and one among the main conclusions was that nanomaterials deserve special attention with regard to planning and conduct of the studies. In particular, comprehensive physicochemical characterization of the materials as well as test item preparation (i.e., nanomaterial dispersion) revealed crucial for the test outcome. Another important aspect discussed was the choice of the test concentrations, which should not be too high either. Further, for assay selection, it has to be noticed that some assays revealed unsuitable in the case of nanomaterials. For instance, bacterial gene mutation assays were regarded rather unuseful for nanomaterial testing since bacterial cell walls might tend to impede the uptake of nanomaterials (Landsiedel et al. 2009). Unfortunately, the Ames test belongs to the most commonly used assays in tier-1 genotoxicity testing of chemicals. In 2013, EURL ECVAM initiated a project which is meant to evaluate the predictivity of the Ames assay and which is expected allowing a better identification of cases in which the Ames test produces irrelevant data. Along with the revision of the guideline of the bacterial reverse mutation test, the applicability domain is to be more precisely specified, including a statement on its suitability for nanomaterial testing.

Another issue in toxicity testing of nanomaterials in general, but also for genotoxicity testing of nanomaterials in particular, is the lack of studies that address a rather large and comprehensive set of nanomaterials (Warheit and Donner 2015). Similarly, Krug concluded that most studies apply only a limited number of nanomaterials but nonetheless have the heart to present rather general and far-reaching conclusions on the particle's safety or hazardous effects-that are, actually, not supported by the data presented (Krug 2014). Having a look at published genotoxicity studies of nanomaterials, indeed hardly any study has used a rather large set of nanomaterials. One of the rare exceptions is the work published by Kermanizadeh and coworkers, where the cytotoxicity and genotoxicity of ten different nanomaterials were assessed in human HK-2 cells (Kermanizadeh et al. 2013). Due to contradicting data on the in vitro genotoxicity of nanomaterials in different studies, and given that no benchmark or reference materials exist, it appears highly advisable to test a large panel of nanomaterials in one and the same study though.

Often it becomes challenging to identify the particular reason(s) why published studies report contradictory results on the same or similar nanomaterials. In some cases, this could be due to the usage of slightly differing 
nanomaterials-although authors may have claimed and actually thought to use identical ones. Upon applying only limited and incomplete material characterization measures, small differences in particle configuration are usually not easy to recognize. In other cases, different dispersion protocols might have been applied or different assays or cell lines have been used. To overcome this hassle, reference or benchmark materials being specific for the respective endpoint would greatly help and facilitate the comparison of different studies.

Now, what exactly is currently known about the genotoxicity of different types of nanomaterials? Are there some common features unraveled yet? The highest commercialized nanomaterials are metals, metal oxides or carbon-based materials. Among the metals, certainly the best-studied kind of material is nanosilver. While several in vitro studies could detect genotoxicity for various types of nanosilver, most in vivo studies failed to do so (reviewed in McShan et al. 2014; Reidy et al. 2013; Klien and Godnic-Cvar 2012). The role of nanosilver dissolution on this outcome is still not fully understood. It is widely assumed that toxicity of nanosilver is mediated by ionic silver. However, particulate silver may also exert adverse effects. For instance, it was shown that nanosilver can directly interact with DNA, thereby altering the DNA structure (McShan et al. 2014). By contrast, studies on other metal nanoparticles, except nanogold, are not as frequent. Nevertheless, available data indicate detectable genotoxicity for cobalt, nickel and copper nanoparticles in vitro and at least for cobalt and nickel also in vivo (Magaye et al. 2012).

Among the group of metal oxides, $\mathrm{ZnO}$ and $\mathrm{TiO}_{2}$ nanomaterials are certainly most important in terms of consumer product applications. While toxicity of $\mathrm{TiO}_{2}$ has been frequently assessed, much less data are available on $\mathrm{ZnO}$. Again, in vitro studies frequently reported positive genotoxicity for both kinds of nanomaterials, while in vivo studies often failed to do so (Chen et al. 2014; Kwon et al. 2014). For some of these metal oxide particles, e.g., $\mathrm{ZnO}$, part of the toxicity will likely be mediated by soluble ions. On the other hand, it has been shown that particle's properties affecting cellular uptake reveal more important in the determination of toxicity including genotoxicity when compared to parameters that only influence solubility (reviewed in Hahn et al. 2014).

For carbon-based materials, most work is focused on carbon nanotubes (CNTs), where plenty of studies have demonstrated a genotoxic potential for both single- and multi-walled CNTs (reviewed in van Berlo et al. 2012). Mechanisms of genotoxicity were often linked to oxidative stress, yet formation of reactive oxygen species (ROS) in cells might also be protective in the case of CNTs due to oxidative biodegradation (Shvedova et al. 2012). In principle, ROS can be directly CNT-induced in the vicinity of cells (e.g., neutrophils), or may arise upon cellular internalization through the interference of CNTs with mitochondrial respiration or via depletion of antioxidant species (reviewed in Manke et al. 2013). In particular, cellular DNA damage can result from direct interaction between CNTs and the genome, or indirectly via CNT-induced oxidative stress and inflammatory responses. Interestingly, at least for multi-walled CNTs, most of the ROS formation seems to result from cellular activation and an involvement of NADPH oxidase and superoxide radicals (Funahashi et al. 2015). This is contrasting the results obtained with asbestos fibers, as do data that provide evidence that CNT fibers may also scavenging ROS to certain extents, an effect that actually should be inversely related to its genotoxicity (van Berlo et al. 2012). Although these effects are currently not fully understood, the assumption that the mechanisms of CNTand asbestos-mediated toxicity might be similar reveals rather premature. Based on current evidence, it occurs that detailed molecular mechanisms differ (Jaurand et al. 2009; Donaldson et al. 2013).

In summary, for toxicity testing of nanomaterials, critical issues have been identified in recent years. In particular, the choice of the cell model and the assay selection deserve special attention. Furthermore, full material characterization, including material characterization under assay conditions, and the kind of preparation of the test item are of utmost importance. In the absence of established benchmark materials, studies assessing a large panel of different nanomaterials should be preferred in hazard characterization. Finally, the issue of test concentration(s) is highly relevant as it clearly contributes to the increase in the number of false positive results. Therefore, relevant test concentrations should be identified and justified through an appropriate in vitro-in vivo extrapolation. By considering all of these issues, the planning and conduct of reliable in vitro genotoxicity studies of nanomaterials will become feasible. The responsibility of scientific journals may remain to identify meaningful results and to extract them from the bulk of studies presenting irrelevant data. A basic set of minimum quality standards is already lying on the table. This is expected to grow fast, in good parts due to the lively discussions on the usefulness of data published in nanosafety research.

\section{References}

Aardema MJ, Barnett BC, Khambatta Z, Reisinger K, OuedraogoArras G, Faquet B, Ginestet AC, Mun GC, Dahl EL, Hewitt NJ, Corvi R, Curren RD (2010) International prevalidation studies of the EpiDerm 3D human reconstructed skin micronucleus (RSMN) assay: transferability and reproducibility. Mutat Res 701:123-131 
Adler S, Basketter D, Creton S, Pelkonen O, van Benthem J, Zuang V, Andersen KE, Angers-Loustau A, Aptula A, Bal-Price A, Benfenati E, Bernauer U, Bessems J, Bois FY, Boobis A, Brandon E, Bremer S, Broschard T, Casati S, Coecke S, Corvi R, Cronin M, Daston G, Dekant W, Felter S, Grignard E, Gundert-Remy U, Heinonen T, Kimber I, Kleinjans J, Komulainen H, Kreiling R, Kreysa J, Leite SB, Loizou G, Maxwell G, Mazzatorta P, Munn S, Pfuhler S, Phrakonkham P, Piersma A, Poth A, Prieto P, Repetto G, Rogiers V, Schoeters G, Schwarz M, Serafimova R, Tähti H, Testai E, van Delft J, van Loveren H, Vinken M, Worth A, Zaldivar JM (2011) Alternative (non-animal) methods for cosmetics testing: current status and future prospects. Arch Toxicol 85:367-485

Chen T, Yan J, Yan L (2014) Genotoxicity of titanium dioxide nanoparticles. J Food Drug Anal 22:95-104

Corvi R, Albertini S, Hartung T, Hoffmann S, Maurici D, Pfuhler S, van Benthem J, Vanparys P (2008) ECVAM retrospective validation of in vitro micronucleus test (MNT). Mutagenesis 23:271-283

Donaldson K, Poland CA, Murphy FA, Mac Farlane M, Chernova T, Schinwald A (2013) Pulmonary toxicity of carbon nanotubes and asbestos-similarities and differences. Adv Drug Deliv Rev 65:2078-2086

Funahashi S, Okazaki Y, Ito D, Asakawa A, Nagai H, Tajima M, Toyokuni S (2015) Asbestos and multi-walled carbon nanotubes generate distinct oxidative responses in inflammatory cells. J Clin Biochem Nutr 56:111-117

Hahn D, Wiemann M, Haase A, Ossig R, Alessandrini F, Ma-Hock L, Landsiedel R, Nern M, Vennemann A, Luch A, Driessen MD, Dopp E, Schnekenburger J (2014) Toxicological effects of metal oxide nanomaterials. In: Wohlleben W, Kuhlbusch T, Lehr CM (eds) Safety of nanomaterials along their lifecycle release, exposure and human hazards. Taylor \& Francis, Oxford

Jaurand MCF, Renier A, Daubriac J (2009) Mesothelioma: do asbestos and carbon nanotubes pose the same health risk? Part Fibre Toxicol 6:16

Kermanizadeh A, Vranic S, Boland S, Moreau K, Baeza-Squiban A, Gaiser BK, Andrzejczuk LA, Stone V (2013) An in vitro assessment of panel of engineered nanomaterials using a human renal cell line: cytotoxicity, pro-inflammatory response, oxidative stress and genotoxicity. BMC Nephrol 14:96-108

Kirkland D, Pfuhler S, Tweats D, Aardema M, Corvi R, Darroudi F, Elhajouji A, Glatt H, Hastwell P, Hayashi M, Kasper P, Kirchner S, Lynch A, Marzin D, Maurici D, Meunier JR, Muller L, Nohynek G, Parry J, Parry E, Thybaud V, Tice R, van Benthem J, Vanparys P, White P (2007) How to reduce false positive results when undertaking in vitro genotoxicity testing and thus avoid unnecessary follow-up animal tests: report of an ECVAM workshop. Mutat Res 628:31-55

Kirkland D, Kasper P, Müller L, Corvi R, Speit G (2008) Recommended lists of genotoxic and non-genotoxic chemicals for assessment of the performance of new or improved genotoxicity tests: a follow-up to an ECVAM workshop. Mutat Res 653:99-108

Klien K, Godnic-Cvar J (2012) Genotoxicity of metal nanoparticles: focus on in vivo studies. Arh Hig Rada Toksikol 63:133-145
Krug HF (2014) Nanosafety research—are we on the right track? Angew Chem Int Ed 53:12304-12319

Kwon JY, Koedrith P, Seo YR (2014) Current investigations into the genotoxicity of zinc oxide and silica nanoparticles in mammalian models in vitro and in vivo: carcinogenic/genotoxic potential, relevant mechanisms and biomarkers, artifacts, and limitations. Int J Nanomed 9(Suppl 2):271-286

Landsiedel R, Kapp MD, Schulz M, Wiench K, Oesch F (2009) Genotoxicity investigations on nanomaterials: methods, preparation and characterization of test material, potential artifacts and limitations-many questions, some answers. Mutat Res 681:241-258

Magaye R, Zhao J, Bowmann L, Ding D (2012) Genotoxicity and carcinogenicity of cobalt-, nickel- and copper-based nanoparticles. Exp Ther Med 4:551-561

Manke A, Wang L, Rojanasakul Y (2013) Mechanisms of nanoparticle-induced oxidative stress and toxicity. BioMed Res Int 2013:942916. doi:10.1155/2013/942916

McShan D, Ray PC, Yu H (2014) Molecular toxicity mechanism of nanosilver. J Food Drug Anal 22:116-127

OECD workshop report on genotoxicity testing of nanomaterials, ENV/JM/MONO(2014)34

Oesch F, Landsiedel R (2012) Genotoxicity investigations on nanomaterials. Arch Toxicol 86:985-994

Parry JM, Parry E, Phrakonkham P, Corvi R (2010) Analysis of published data for top concentration considerations in mammalian cell genotoxicity testing. Mutagenesis 25:531-538

Pfuhler S, Elespuru R, Aardema MJ, Doak SH, Donner M, Honma M, Kirsch-Volders M, Landsiedel R, Manjanatha M, Singer T, Kim JH (2013) Genotoxicity of nanomaterials: refining strategies and tests for hazard identification. Environ Mol Mutagen 54:229-239

Reidy B, Haase A, Luch A, Dawson KA, Lynch I (2013) Mechanisms of silver nanoparticle release, transformation and toxicity: a critical review of current knowledge and recommendations for future studies and applications. Materials 6:2295-2350

Reus AA, Reisinger K, Downs TR, Carr GJ, Zeller A, Corvi R, Krul CAM, Pfuhler S (2013) Comet assay in reconstructed 3D human epidermal skin models-investigation of intra- and interlaboratory reproducibility with coded chemicals. Mutagenesis 28:709-720

Shvedova AA, Kapralov AA, Feng WH, Kisin ER, Murray AR, Mercer RR, St. Croix CM, Lang MA, Watkins SC, Konduru NV, Allen BL, Conroy J, Kotchey GP, Mohamed BM, Meade AD, Volkov Y, Star A, Fadeel B, Kagan VE (2012) Impaired clearance and enhanced pulmonary inflammatory/fibrotic response to carbon nanotubes in myeloperoxidase-deficient mice. PLoS One 7:e30923

van Berlo D, Clift MJD, Albrecht C, Schins RPF (2012) Carbon nanotubes: an insight into the mechanisms of their potential genotoxicity. Swiss Med Wkly 142:w13698

Warheit DB, Donner EM (2015) How meaningful are risk determinations in the absence of a complete dataset? Making the case for publishing standardized test guidelines and "no effect" studies for evaluating the safety of nanoparticulates versus spurious "high effect" results from single investigative studies. Sci Technol Adv Mater 16:3 